\title{
Electropolishing of Additive Manufactured 17-4 PH Stainless Steel Using Sulfuric Acid
}

\author{
Jun-Kai Chang ${ }^{1,2}$, Chia-Yu Lee ${ }^{3}$, Yu-Chih Tzeng ${ }^{4,7}$, Ming-Hsien Lin ${ }^{5,7}$, Ming-Der Ger ${ }^{5,7}$, \\ Chun-Hsiang Kao ${ }^{6}$, Chih-Peng Chen ${ }^{6}$, Kuo-Kuang Jen ${ }^{6}$, Shun-Yi Jian ${ }^{5,7, *}$ \\ ${ }^{1}$ Research and Development Department, Chung Yo Materials Corporation, Gangshan district, \\ Kaohsiung City 820, Taiwan \\ ${ }^{2}$ Laser and Additive Manufacturing Technology Center, Industrial Technology Research Institute, Liujia \\ district, Tainan City 734, Taiwan \\ ${ }^{3}$ Graduate School of Defense Science, Chung Cheng Institute of Technology, National Defense \\ University, Taoyuan, Taiwan \\ ${ }^{4}$ Department of Power Vehicle and Systems Engineering, Chung Cheng Institute of Technology, \\ National Defense University, Dasi, Taoyuan 335, Taiwan \\ ${ }^{5}$ Department of Chemical \& Materials Engineering, Chung Cheng Institute of Technology, National \\ Defense University, Dasi district, Taoyuan City 335, Taiwan \\ ${ }^{6}$ Missile and rocket systems research division, National Chung-Shan Institute of Science and \\ Technology, Taoyuan City 32546, Taiwan \\ ${ }^{7}$ System Engineering and Technology Program, National Chiao Tung University, Hsinchu City 300, \\ Taiwan \\ *E-mail: ftvko@yahoo.com.tw (S. Y. Jian)
}

doi: $10.20964 / 2021.03 .09$

Received: 1 November 2020 / Accepted: 21 December 2020 / Published: 31 January 2021

The sulfuric acid electropolishing system was built to reduce the surface roughness of the additivemanufactured 17-4 PH stainless steel. The extra $10 \mathrm{~V}$ power was applied to electropolish the AM specimen to reach the desired surface roughness. The roughness was reduced to $6.76 \mu \mathrm{m}$ and additional corrosion resistance was given. The corrosion potential became noble and the corrosion current density decreased to approximately $10^{-6} \mathrm{~A} / \mathrm{cm}^{2}$. The post treatment of the electropolishing process accurately improved both the surface roughness and the corrosion resistance, demonstrating the electropolish process is a useful application in AM objects.

Keywords: Additive Manufacturing; Electropolish; 17-4 PH stainless steel; Roughness 
(C) 2021 The Authors. Published by ESG (www.electrochemsci.org). This article is an open access article distributed under the terms and conditions of the Creative Commons Attribution license (http://creativecommons.org/licenses/by/4.0/). 